\title{
ON THE CONSTRUCTION OF THE IRREDUCIBLE REPRESENTATIONS OF THE HYPERALGEBRA OF A UNIVERSAL CHEVALLEY GROUP
}

\author{
HENRY NIEMI
}

1. Introduction. Let $K$ be an algebraically closed field of characteristic $p>3$. Let $\mathfrak{g}$ be a finite dimensional complex semisimple Lie algebra and $G$ the universal Chevalley group of type $g$ over $K$. If $U_{Z}$ denotes Kostant's $Z$-form of the universal enveloping algebra of $\mathfrak{g}$, then the infinite dimensional associative $K$-algebra $U_{K}=$ $U_{Z} \otimes_{Z} K$ is known as the hyperalgebra of $G$ ([2], [6]).

Each finite dimensional irreducible rational $G$-module is known to admit the structure of a $U_{K}$-module and vice versa ([2], [5]). Moreover, for each positive integer $\boldsymbol{r}$ the algebra $U_{K}$ contains a finite dimensional subalgebra $\boldsymbol{u}_{\boldsymbol{r}}$ in such a way that finite dimensional irreducible $\boldsymbol{u}_{\boldsymbol{r}}$-modules correspond one-to-one to finite dimensional irreducible $G_{r}$-modules over $K$, where $G_{r}$ is the group of points in $G$ rational over the finite field of $p^{r}$ elements.

In [10] it was observed that the algebras $U_{K}$ and $\boldsymbol{u}_{\boldsymbol{r}}$ all admit a s.c. good triangular decomposition. Using this we show how to explicitly construct the finite dimensional irreducible $U_{K}$ - and $\boldsymbol{u}_{r}$-modules starting from certain one-dimensional representations of a suitable subalgebra. This provides a new direct way of realizing and classifying all irreducible $U_{K^{-}}$and $\boldsymbol{u}_{\boldsymbol{r}}$-modules, without relying on the classical highest weight theory of $G$-modules as in [6]. Moreover, the finite dimensional irreducible $U_{K}$-modules are shown to have a certain tensor product decomposition (Proposition 6). When interpreted as a result of $G$-modules this provides a new proof for the celebrated Steinberg's tensor product theorem.

2. Algebras with good triangular decomposition. Let $A$ be an associative algebra with 1 over a field $K$. Let $B$ be a subalge bra of $A(1 \in B)$ and assume $B=H \oplus R$, a vector space direct sum, where $H$ is a subalgebra of $A(1 \in H)$ and $R$ a two-sided ideal of $B$. Let us assume furthermore that $R$ acts nilpotently on every irreducible finite dimensional left $A$-module. As in [10], we say that $A$ admits a triangular decomposition over $B$ if there exists a left $B$-, right $H$-module homomorphism $\gamma: A \rightarrow B$ such that $\left.\gamma\right|_{B}=1_{B}$. In this case $A=H \oplus R \oplus \operatorname{ker}(\gamma)$.

If $M$ is an arbitrary left $A$-module let $M^{\operatorname{ker}(\gamma)}=\{m \in M \mid \operatorname{ker}(\gamma) m=0\}$. Then $M^{\text {ker( }(\gamma)}$ is a left $H$-module. Supposing $A$ admits a triangular decomposition over 
$B$ and $M^{\mathrm{ker}(\gamma)} \neq 0$ for every finite dimensional left $A$-module $M \neq 0$, then $A$ is said to admit a good triangular decomposition over $B$.

Suppose now that $A$ admits a triangular decomposition over $B$ via $\gamma$. Then any left $H$-module $W$ becomes a left $B$-module by way of

$$
(h+r) v=h v \quad \text { for every } h \in H, \quad r \in R, \quad v \in W .
$$

Furthermore, $P(W)=\operatorname{Hom}_{B}(A, W)$ is viewed as a left $A$-module in the usual fashion: $(a \cdot f)\left(a^{\prime}\right)=f\left(a^{\prime} a\right)$ for every $a, a^{\prime} \in A, f \in P(W)$. Finally the map $\omega: W \rightarrow P(W)$, given by $\omega(v)(a)=\gamma(a) v, v \in W, a \in A$, is an injective left $H$-module map. We denote by $W^{\prime}=A \omega(W)$ the left $A$-submodule of $P(W)$ generated by $\omega(W)$.

Remark. If $\operatorname{dim} A=\infty$, the module $W^{\prime}$ may sometimes be infinite dimensional even though $\operatorname{dim} W<\infty$. In the sequel all modules and representations are automatically assumed to be finite dimensional.

An argument, similar to those in [10] and [13], yields the following result.

Proposition 1. Assume $A$ admits a good triangular decomposition over B via $\gamma$ and that $\operatorname{ker}(\gamma) \operatorname{ker}(\gamma) \subseteq \operatorname{ker}(\gamma)$. Then the following are true:

(i) $W_{1} \cong{ }_{H} W_{2}$ if and only if $W_{1}^{\prime} \cong{ }_{A} W_{2}^{\prime}$.

(ii) $\left(W^{\prime}\right)^{\mathrm{ker}(\gamma)}=\omega(W) \cong{ }_{H} W$ for each left $H$-module $W$.

(iii) If $W$ is an irreducible left $H$-module and $\operatorname{dim} W^{\prime}<\infty$, then $W^{\prime}$ is an irreducible left A-module.

(iv) If $M$ is an irreducible left $A$-module, then $M^{\mathrm{ker}(\gamma)}$ is an irreducible left $H$-module and $M \cong{ }_{A}\left(M^{\mathrm{ker}(\gamma)}\right)^{\prime}$.

Let $\operatorname{Irr}(A)$ (resp. Irr $(H))$ denote the set of isomorphism classes of irreducible left $A$-modules (resp. $H$-modules). Proposition 1 established a bijective correspondence between the sets $\operatorname{Irr}(A)$ and $\left\{[W] \in \operatorname{Irr}(H) \mid \operatorname{dim} W^{\prime}<\infty\right\}$ where $[W]$ denotes the class of $H$-modules isomorphic to $W$. If $\operatorname{dim} A<\infty$ this latter set is of course equal to $\operatorname{Irr}(H)$.

3. The algebras $U_{K}$ and $\boldsymbol{u}_{\boldsymbol{r}}$. Let $\mathfrak{h}$ be a maximal torus in the semisimple Lie algebra $\mathfrak{g}$, and $\Phi$ the root system of $\mathfrak{g}$ with respect to $\mathfrak{h}$. Let $\Delta=\left\{\alpha_{1}, \ldots, \alpha_{l}\right\}$ denote a basis of $\Phi$ and $\Phi^{+}=\left\{\alpha_{1}, \ldots, \alpha_{m}\right\}, \Phi^{-}=-\Phi^{+}$, the sets of positive (resp. negative) roots with respect to $\Delta$. We fix a Chevalley basis $\left\{x_{\alpha}, \alpha \in \Phi ; h_{i}=h_{\alpha_{i}}(i=1, \ldots, l)\right\}$ for $\mathfrak{g}$ and let $U_{Z}$ denote Kostant's $Z$-form of $U(\mathfrak{g})$, i.e. the subring generated by all $x_{\alpha}^{t} / t !, \alpha \in \Phi, t \in Z^{+}$.

From now on let $K$ be an algebraically closed field of characteristic $p>3$. Set $X_{\alpha, n}=x_{\alpha}^{n} / n ! \otimes 1\left(\alpha \in \Phi, n \in Z^{+}\right)$and

The products

$$
H_{i, b}=\left(\begin{array}{c}
h_{i} \\
b
\end{array}\right) \otimes 1 \quad\left(i=1, \ldots, l, b \in Z^{+}\right) .
$$

$$
\left\{\prod_{i=1}^{m} X_{-\alpha_{i}, a_{i}} \prod_{i=1}^{l} H_{i, b_{i}} \prod_{i=1}^{m} X_{\alpha_{i}, c_{i}} \mid a_{i}, b_{i}, c_{i} \in \mathbf{Z}^{+}\right\}
$$


are known to form a $K$-basis for the $K$-algebra $U_{K}=U_{Z} \otimes_{Z} K$, which is called the hyperalgebra.

If $S$ is any subset of $U_{K}$ let $\langle S\rangle$ denote the subalgebra of $U_{K}$ generated by $S$. For any $r \in N$ let $\boldsymbol{u}_{\boldsymbol{r}}=\left\langle\left\{X_{\alpha, t} \mid \alpha \in \Phi, 0 \leqq t<p^{r}\right\}\right\rangle$. It was shown in [6] that the products

$$
\left\{\prod_{i=1}^{m} X_{-\alpha_{i}, a_{i}} \prod_{i=1}^{l} H_{i, b_{i}} \prod_{i=1}^{m} X_{\alpha_{i}, c_{i}} \mid 0 \leqq a_{i}, b_{i}, c_{i}<p^{r}\right\}
$$

form a $K$-basis for $\boldsymbol{u}_{\boldsymbol{r}}$. Moreover $X_{\alpha, t}^{p}=0$ and $H_{i, b}^{p}=H_{i, b}$ whenever $\alpha \in \Phi, t \in N$, $i=1, \ldots, l$ and $b \in \boldsymbol{Z}^{+}$. The following subalgebras are also needed

$$
\begin{aligned}
& \boldsymbol{h}_{\boldsymbol{r}}=\left\langle\left\{H_{i, b} \mid 1 \leqq i \leqq l, 0 \leqq b<p^{r}\right\}\right\rangle, \\
& \boldsymbol{x}_{\boldsymbol{r}}=\left\langle\left\{X_{\alpha, c} \mid \alpha \in \Phi^{+}, 0<c<p^{r}\right\}\right\rangle, \\
& \boldsymbol{y}_{\boldsymbol{r}}=\left\langle\left\{X_{-\alpha, a} \mid \alpha \in \Phi^{+}, 0<a<p^{r}\right\}\right\rangle, \\
& \boldsymbol{b}_{\boldsymbol{r}}=\left\langle\boldsymbol{h}_{\boldsymbol{r}} \cup \boldsymbol{y}_{r}\right\rangle .
\end{aligned}
$$

Lemma 1. The algebras $\boldsymbol{h}_{\boldsymbol{r}}, \boldsymbol{x}_{\boldsymbol{r}}$ and $\boldsymbol{y}_{\boldsymbol{r}}$ are already generated as algebras by the sets $\left\{1, H_{i, p^{j}} \mid 1 \leqq i \leqq l, 0 \leqq j \leqq r-1\right\},\left\{X_{\alpha, p^{j}} \mid \alpha \in \Delta, 0 \leqq j \leqq r-1\right\}$ and $\left\{X_{-\alpha, p^{j}} \mid \alpha \in \Delta, 0 \leqq j \leqq r-1\right\}$ respectively.

Proof. For $\boldsymbol{h}_{\boldsymbol{r}}$ this follows directly from the proof of Proposition 2.1 in [6]. In case of $x_{r}$ let $\alpha \in \Phi^{+}$and $0<c<p^{r}$. Using induction with respect to $c$, it can easily be shown that $X_{\alpha, c} \in\left\langle\left\{X_{\alpha, p^{j}} \mid 0 \leqq j \leqq r-1\right\}\right\rangle$. Since $p>3$ the proof of Proposition (7I) in [8] implies that $X_{\alpha, p^{j}} \in\left\langle\left\{X_{\alpha, p^{j}} \mid \alpha \in \Delta, 0 \leqq j \leqq r-1\right\}\right\rangle$ for all $\alpha \in \Phi^{+}, 0 \leqq j \leqq$ $r-1$. This proves the assertion about $\boldsymbol{x}_{\boldsymbol{r}}$, and $\boldsymbol{y}_{\boldsymbol{r}}$ can be handled analogously.

Lemma 2. Let $\alpha \in \Phi, i \in\{1, \ldots, l\}, a, c, k, s \in \boldsymbol{Z}^{+}$and $r \in \boldsymbol{N}$. Let $\beta \in \Phi$ be such that $\alpha \neq-\beta$ and $\alpha+\beta \notin \Phi$. Then the following commutation rules hold:

$$
\begin{gathered}
X_{\alpha, c} X_{-\alpha, a}=\sum_{k=0}^{\min (a, c)} X_{-\alpha, a-k}\left\{\left(\begin{array}{c}
h_{\alpha}-a-c+2 k \\
k
\end{array}\right) \otimes 1\right\} X_{\alpha, c-k}, \\
H_{i, a} X_{\alpha, c}=X_{\alpha, c}\left\{\left(\begin{array}{c}
h_{i}+c \alpha\left(h_{i}\right) \\
a
\end{array}\right) \otimes 1\right\}, \\
X_{\alpha, c} H_{i, a}=\left\{\left(\begin{array}{c}
h_{i}-c \alpha\left(h_{i}\right) \\
a
\end{array}\right) \otimes 1\right\} X_{\alpha, c}, \\
X_{\alpha, k} X_{\beta, s}=X_{\beta, s} X_{\alpha, k} \\
X \boldsymbol{x}_{r}=\boldsymbol{x}_{r} X, Y \boldsymbol{y}_{r}=\boldsymbol{y}_{r} Y, \text { where } X=\bigcup_{r=1}^{\infty} \boldsymbol{x}_{r}, Y=\bigcup_{r=1}^{\infty} \boldsymbol{y}_{r} .
\end{gathered}
$$

Proof. The identities (4)-(6) are well known ([4]). Because of our assumptions, $\left[x_{\alpha}, x_{\beta}\right]=0$ and from this (7) follows immeciiately. To prove the commutation rule $X \boldsymbol{x}_{\boldsymbol{r}}=\boldsymbol{x}_{\boldsymbol{r}} X$ we first prove $X \boldsymbol{x}_{\boldsymbol{r}} \subseteq \boldsymbol{x}_{\boldsymbol{r}} X$. It suffices to show that $X_{\alpha, k} X_{\beta, s} \in \boldsymbol{x}_{\boldsymbol{r}} X$ for all $\alpha, \beta \in \Phi^{+}, k \in N$ and $0<s<p^{r}$. If $\alpha+\beta \notin \Phi$ then by (7) $X_{\alpha, k} X_{\beta, s}=X_{\beta, s} X_{\alpha, k} \in x_{r} X$. We suppose now that $\alpha+\beta \in \Phi$. Let $U_{K}[[t, u]]$ be the ring of formal power series over 
$U_{K}$, where $t$ and $u$ are independent variables. Let $x_{\gamma}(t)=\sum_{n \geqq 0} t^{n} X_{\gamma, n} \in U_{K}[[t, u]]$, where $\gamma \in \Phi$. Then there are integers $c_{i j}$ such that

$$
x_{\alpha}(t) x_{\beta}(u)=\prod_{i, j \geqq 1} x_{i \alpha+j \beta}\left(c_{i j} t^{i} u^{j}\right) x_{\beta}(u) x_{\alpha}(t)
$$

([11], p. 22). Comparing the coefficients of the term $t^{k} u^{s}$ in the above equation we see that $X_{\alpha, k} X_{\beta, s} \in \boldsymbol{x}_{r} X$. The inclusion $\boldsymbol{x}_{r} X \subseteq X \boldsymbol{x}_{r}$ can be proved analogously. The commutation rule $Y \boldsymbol{y}_{r}=\boldsymbol{y}_{r} Y$ is handled similarly.

Set $\boldsymbol{r}_{\boldsymbol{r}}=\boldsymbol{y}_{\boldsymbol{r}} \boldsymbol{h}_{\boldsymbol{r}}$. Lemma 2 implies $\boldsymbol{y}_{\boldsymbol{r}} \boldsymbol{h}_{\boldsymbol{r}}=\boldsymbol{h}_{\boldsymbol{r}} \boldsymbol{y}_{\boldsymbol{r}}$, whence $\boldsymbol{r}_{\boldsymbol{r}}$ is a two-sided ideal of $\boldsymbol{b}_{\boldsymbol{r}}$. It is not difficult to see that the algebras $\boldsymbol{x}_{\boldsymbol{r}}, \boldsymbol{y}_{\boldsymbol{r}}$ and $\boldsymbol{r}_{\boldsymbol{r}}$ are all nilpotent. Write

$$
\boldsymbol{u}_{r}=\boldsymbol{b}_{r} \oplus \boldsymbol{b}_{r} \boldsymbol{x}_{\boldsymbol{r}}=\boldsymbol{h}_{\boldsymbol{r}} \oplus \boldsymbol{r}_{r} \oplus \boldsymbol{b}_{\boldsymbol{r}} \boldsymbol{x}_{\boldsymbol{r}}
$$

and let $\gamma_{r}: \boldsymbol{u}_{\boldsymbol{r}} \rightarrow \boldsymbol{b}_{\boldsymbol{r}}$ be the projection onto the first factor. The algebra $\boldsymbol{u}_{\boldsymbol{r}}$ now admits a good triangular decomposition over $\boldsymbol{b}_{r}$ with respect to $\gamma_{\boldsymbol{r}}$ (cf. [10]). Moreover, $\operatorname{ker}\left(\gamma_{r}\right) \operatorname{ker}\left(\gamma_{r}\right) \subseteq \operatorname{ker}\left(\gamma_{r}\right)$.

Similarly, if we set $H=\bigcup_{r=1}^{\infty} \boldsymbol{h}_{\boldsymbol{r}}, B=\cup_{\boldsymbol{r}=1}^{\infty} \boldsymbol{b}_{\boldsymbol{r}}$ and $R=\cup_{\boldsymbol{r}=1}^{\infty} \boldsymbol{r}_{\boldsymbol{r}}$ then $R=Y H=$ $=H Y$ and

$$
U_{K}=B \oplus B X=H \oplus R \oplus B X .
$$

The hyperalgebra $U_{K}$ now admits a good triangular decomposition over $B$ with respect to the projection $\gamma: U_{K} \rightarrow B$ and $\operatorname{ker}(\gamma) \operatorname{ker}(\gamma) \leqq \operatorname{ker}(\gamma)$.

4. The irreducible representations of $U_{K}$ and $\boldsymbol{u}_{r}$. We have seen above that the hyperalgebra $U_{K}$ and each of its finite dimensional subalgebras $\boldsymbol{u}_{\boldsymbol{r}}$ satisfy the hypotheses in Proposition 1. Hence the irreducible $U_{K}$ - and $\boldsymbol{u}_{r}$-modules can all be obtained from irreducible $H$ - and $\boldsymbol{h}_{\boldsymbol{r}}$-modules respectively using the lifting process of $\S 2$. The question now arises: which irreducible $H$-modules yield finite dimensional $U_{K}$-modules?

The algebras $H$ and $\boldsymbol{h}_{r}$ are commutative. The set $m_{r}=\left\{\prod_{i=1}^{l} H_{i, b_{i}} \mid 0 \leqq b_{i}<p^{r}\right\}$ is a $K$-basis for $\boldsymbol{h}_{r}$ and $M=\bigcup_{r=1}^{\infty} m_{r}$ a $K$-basis for $H$. Since $H_{i, b}^{p}=H_{i, b}$ for all $i$ and $b$ the same argument as in [7], p. 193, shows that all $H$ - and $\boldsymbol{h}_{\boldsymbol{r}}$-modules are completely reducible. Irreducible representations of $H$ and $\boldsymbol{h}_{r}$ are naturally onedimensional, let $\varphi: H \rightarrow K, \varphi_{r}: \boldsymbol{h}_{\boldsymbol{r}} \rightarrow K$ be any such. Finally, let $W(\varphi)=K w_{\varphi}$ and $W\left(\varphi_{r}\right)=K w_{\varphi_{r}}$ denote the one-dimensional modules corresponding to these algebra homomorphisms.

If $\lambda_{1}, \ldots, \lambda_{l}$ are the fundamental dominant weights, $P=\boldsymbol{Z} \lambda_{1} \oplus \ldots \oplus \boldsymbol{Z} \lambda_{l}$ the set of all weights, then the weights in $P_{p^{r}}=\left\{\sum_{i=1}^{l} m_{i} \lambda_{i} \mid 0 \leqq m_{i}<p^{r}\right\}$ are called restricted and those in $P^{+}=\bigcup_{r=1}^{\infty} P_{p^{r}}$ dominant. Each $\lambda \in P$ gives rise to an algebra homomorphism

$$
\varphi_{\lambda}: H \rightarrow K, \varphi_{\lambda}\left(H_{i, b}\right)=\left(\begin{array}{c}
\lambda\left(h_{i}\right) \\
b
\end{array}\right) \text { for all } i=1, \ldots, l, b \in \boldsymbol{Z}^{+}
$$

and one knows that $\varphi_{\lambda} \neq \varphi_{\mu}$ whenever $\lambda \neq \mu$. For each $\lambda \in P_{p^{r}}$ let $\varphi_{\lambda} \mid \boldsymbol{h}_{\boldsymbol{r}}=\varphi_{\boldsymbol{r}, \lambda}$, a one-dimensional representation of $\boldsymbol{h}_{\boldsymbol{r}}$. Let us also use the following shorter nota- 
tions $W(r, \lambda)=W\left(\varphi_{r, \lambda}\right)=K w_{r, \lambda}$ and $\quad V(r, \lambda)=W(r, \lambda)^{\prime}$ whenever $\lambda \in P_{p^{r}}$. It was shown in [6] that the representations $\varphi_{r, \lambda}$ are pairwise non-equivalent and constitute all algebra homomorphisms $\boldsymbol{h}_{\boldsymbol{r}} \rightarrow K$. These observations together with Proposition 1 prove

Proposition 2. If $\lambda, \mu \in P_{p^{r}}, \lambda \neq \mu$, then the irreducible $\boldsymbol{u}_{r}$-modules $V(r, \lambda)$ and $V(r, \mu)$ are mutually non-isomorphic. In fact $\operatorname{Irr}\left(\boldsymbol{u}_{r}\right)=\left\{[V(r, \lambda)] \mid \lambda \in P_{p^{r}}\right\}$.

According to Proposition $1 \operatorname{Irr}\left(U_{K}\right)=\left\{\left[W(\varphi)^{\prime}\right] \mid \operatorname{dim} W(\varphi)^{\prime}<\infty\right\}$. We shall show next that $\left\{\varphi \mid \operatorname{dim} W(\varphi)^{\prime}<\infty\right\}=\left\{\varphi_{\lambda} \mid \lambda \in P^{+}\right\}$, and thus that $\operatorname{Irr}\left(U_{K}\right)=\left\{\left[W(\lambda)^{\prime}\right] \mid \lambda \in P^{+}\right\}$, where $W(\lambda)=W\left(\varphi_{\lambda}\right)=K w_{\lambda}$. The equality $\operatorname{Irr}\left(U_{K}\right)=\bigcup_{r=1}^{\infty} \operatorname{Irr}\left(\boldsymbol{u}_{r}\right)$ then also follows.

Lemma 3. $\left\{\varphi \mid \operatorname{dim} W(\varphi)^{\prime}<\infty\right\} \subseteq\left\{\varphi_{\lambda} \mid \lambda \in P^{+}\right\}$.

Proof. Assuming $\operatorname{dim} W(\varphi)^{\prime}<\infty$ we first establish the existence of a $b \in N$ such that $\varphi\left(H_{i, a}\right)=0$ whenever $a>b, i=1, \ldots, l$. Suppose this were not the case. There would then exist an index $i \in\{1, \ldots, l\}$ and an infinite sequence of integers $b_{1}<b_{2}<\ldots$ such that $\varphi\left(H_{i, b_{j}}\right) \neq 0$ for all $j \in N$. Let $\omega: W(\varphi) \rightarrow P(W(\varphi))$ be as in $\S 2$. Using the commutation rule (4) one shows readily that the elements $X_{-\alpha_{i}, b_{j}} \omega\left(w_{\varphi}\right) \in W(\varphi)^{\prime}, j \in N$, are all linearly independent. This, however, contradicts the assumption $\operatorname{dim} W(\varphi)^{\prime}<\infty$.

We have now seen that $\varphi\left(M \backslash m_{r}\right)=0$ for some $r \in N$. On the other hand $\varphi$ induces a representation for the subalgebra $\boldsymbol{h}_{\boldsymbol{r}}$ so that $\varphi\left|\boldsymbol{h}_{\boldsymbol{r}}=\varphi_{\lambda}\right| \boldsymbol{h}_{\boldsymbol{r}}$ for some $\lambda \in \boldsymbol{P}_{p^{r}}$. Then $\varphi_{\lambda}\left(M \backslash m_{r}\right)=0$ and $\left.\varphi\right|_{m_{r}}=\left.\varphi_{\lambda}\right|_{m_{r}}$ hence $\varphi=\varphi_{\lambda}$ since they agree on the basis $M$.

Lemma 4. If $i \in\{1, \ldots, l\}$ and $\lambda \in P^{+}$then $X_{-\alpha_{i}, a} \omega\left(w_{\lambda}\right)=0$ for all $a>\lambda\left(h_{i}\right)$.

Proof. According to Proposition $1\left(W(\lambda)^{\prime}\right)^{\operatorname{ker}(\gamma)}=\omega(W(\lambda))=\omega\left(K w_{\lambda}\right)=K \omega\left(w_{\lambda}\right)$. Now $K \omega\left(w_{\lambda}\right) \cap Y \omega\left(w_{\lambda}\right)=0$ since $\omega\left(w_{\lambda}\right)(1)=\gamma(1) w_{\lambda}=w_{\lambda}$ and $\quad\left(y \omega\left(w_{\lambda}\right)\right)(1)=$ $\gamma(y) w_{\lambda}=y w_{\lambda}=0$ for all $y \in Y$. Because $X_{-\alpha_{i}, a} \omega\left(w_{\lambda}\right) \in Y \omega\left(w_{\lambda}\right)$ it suffices to prove that $X_{-\alpha_{i}, a} \omega\left(w_{\lambda}\right) \in\left(W(\lambda)^{\prime}\right)^{\operatorname{ker}(\gamma)}$ whenever $a>\lambda\left(h_{i}\right)$. Furthermore, since $\operatorname{ker}(\gamma)=$ $B X$ and $X=\left\langle\left\{X_{\alpha_{j}, c} \mid j=1, \ldots, l, c \in N\right\}\right\rangle$ (Lemma 1), it suffices to prove that $X_{\alpha_{j}, c} X_{-\alpha_{i}, a} \omega\left(w_{\lambda}\right)=0$ for all $j \in\{1, \ldots, l\}, c \in N, a>\lambda\left(h_{i}\right)$. Using the commutation rules of Lemma 2 this follows by induction on $a$.

Proposition 3. $\left\{\varphi \mid \operatorname{dim} W(\varphi)^{\prime}<\infty\right\}=\left\{\varphi_{\lambda} \mid \lambda \in P^{+}\right\}$.

Proof. It suffices to show $\operatorname{dim} W(\lambda)^{\prime}<\infty$ for all $\lambda \in P^{+}$(Lemma 3). To this end fix $\lambda \in P^{+}=\bigcup_{s=1}^{\infty} P_{p^{s}}$ and assume $\lambda \in P_{p^{r}}$. If we can establish

$$
W(\lambda)^{\prime}=\boldsymbol{u}_{\boldsymbol{r}} \omega(W(\lambda))=\boldsymbol{u}_{r} \omega\left(w_{\lambda}\right)
$$

we are done. The proof of this can be reduced to showing that $M=\boldsymbol{u}_{r} \omega\left(w_{\lambda}\right)$ is a $U_{K}$-submodule of $W(\lambda)^{\prime}$ and this follows if $X M \subseteq M$ and $Y M \subseteq M$. 
Now $M=K \omega\left(w_{\lambda}\right) \oplus \boldsymbol{y}_{r} \omega\left(w_{\lambda}\right)$. First of all $X \omega\left(w_{\lambda}\right)=0$. Using Lemmas 1 and 2 one sees easily that $X \boldsymbol{y}_{r} \subseteq \boldsymbol{u}_{r}+\boldsymbol{u}_{r} X$. Hence

$$
X M=X \boldsymbol{y}_{\boldsymbol{r}} \omega\left(w_{\lambda}\right) \subseteq \boldsymbol{u}_{r} \omega\left(w_{\lambda}\right)=M .
$$

Since $Y=\left\langle\left\{X_{-\alpha_{i}, a} \mid i=1, \ldots, l, a \in N\right\}\right\rangle$ (Lemma 1) Lemma 4 says that $Y \omega\left(w_{\lambda}\right) \subseteq$ $\boldsymbol{y}_{\boldsymbol{r}} \omega\left(w_{\lambda}\right)+Y \boldsymbol{y}_{\boldsymbol{r}} \omega\left(w_{\lambda}\right)$. The algebra $\boldsymbol{y}_{\boldsymbol{r}}$ is nilpotent and $Y \boldsymbol{y}_{\boldsymbol{r}}=\boldsymbol{y}_{\boldsymbol{r}} Y$ by Lemma 2, hence $Y \omega\left(w_{\lambda}\right) \subseteq \boldsymbol{y}_{\boldsymbol{r}} \omega\left(w_{\lambda}\right)$. This implies

$$
Y M=Y\left(K \omega\left(w_{\lambda}\right)+\boldsymbol{y}_{\boldsymbol{r}} \omega\left(w_{\lambda}\right)\right) \subseteq \boldsymbol{y}_{\boldsymbol{r}} \omega\left(w_{\lambda}\right)=M .
$$

Now $M$ is a finite dimensional $U_{K}$-submodule of $W(\lambda)^{\prime}$ and $U_{K}$ admits a good triangular decomposition over $B$ via $\gamma$. Therefore $M^{\mathrm{ker}(\gamma)} \neq 0$. According to Proposition 1

$$
0 \neq M^{\operatorname{ker}(\gamma)} \subseteq\left(W(\lambda)^{\prime}\right)^{\mathbf{k e r}(\gamma)}=\omega(W(\lambda))=K \omega\left(w_{\lambda}\right) .
$$

Thus $M^{\operatorname{ker}(\gamma)}=K \omega\left(w_{\lambda}\right)$ and it follows that $W(\lambda)^{\prime}=U_{K} \omega\left(w_{\lambda}\right)=U_{K} M^{\operatorname{ker}(\gamma)} \subseteq M$. This means $W(\lambda)^{\prime}=M=\boldsymbol{u}_{\boldsymbol{r}} \omega\left(w_{\lambda}\right)$ and the proof is complete.

Proposition 4. If $\lambda, \mu \in P^{+}, \lambda \neq \mu$, then the irreducible $U_{K}$-modules $W(\lambda)^{\prime}$ and $W(\mu)^{\prime}$ are mutually non-isomorphic. In fact $\operatorname{Irr}\left(U_{K}\right)=\left\{\left[W(\lambda)^{\prime}\right] \mid \lambda \in P^{+}\right\}$.

Proof. This is an immediate consequence of Propositions 1 and 3.

For $\lambda \in P^{+}$let $v_{\lambda}=\omega\left(w_{\lambda}\right)$ and $V(\lambda)=W(\lambda)^{\prime}=K v_{\lambda} \oplus Y v_{\lambda}$. The vectors

$$
X_{-\alpha_{1}, a_{1}} \ldots X_{-\alpha_{m}, a_{m}} v_{\lambda} \quad\left(a_{i} \in \boldsymbol{Z}^{+}, i=1, \ldots, m\right)
$$

span the vector space $V(\lambda)$. Because of (5) we get

$$
h X_{-\alpha_{1}, a_{1}} \ldots X_{-\alpha_{m}, a_{m}} v_{\lambda}=\varphi_{\mu}(h) X_{-\alpha_{1}, a_{1}} \ldots X_{-\alpha_{m}, a_{m}} v_{\lambda},
$$

$\mu=\lambda-\sum_{j=1}^{m} a_{j} \alpha_{j}$, for all $a_{i} \in \boldsymbol{Z}^{+}(i=1, \ldots, m), h \in H$. This implies that $V(\lambda)$ has a weight space decomposition

$$
V(\lambda)=\oplus \sum_{\mu \in P(\lambda)} V(\lambda)_{\mu}
$$

where $V(\lambda)_{\mu}=\left\{v \in V(\lambda) \mid h v=\varphi_{\mu}(h) v\right.$ for all $\left.h \in H\right\}$ and $P(\lambda)=\left\{\mu \in P \mid V(\lambda)_{\mu} \neq 0\right\}$ is the set of weights of $V(\lambda)$ with respect to $H$. Formula (11) means that $P(\lambda) \subseteq$ $\left\{\lambda-\sum_{j=1}^{m} a_{j} \alpha_{j} \mid a_{j} \in \boldsymbol{Z}^{+}\right\}$. If $W$ is any $H$-submodule of $V(\lambda)$ then (cf. [8], p. 4)

$$
W=\oplus \sum_{\mu \in P(\lambda)}\left(V(\lambda)_{\mu} \cap W\right) .
$$

Lemma 5. If $\lambda \in P_{p^{r}}, \alpha \in \Delta$ and $\lambda-i \alpha \in P(\lambda)$ then $i \leqq p^{r}-1$.

Proof. Among the weight vectors (10) only $X_{-\alpha, i} v_{\lambda}$ is of weight $\lambda-i \alpha$. Hence Lemma 5 follows from Lemma 4.

Proposition 5. Considered as $\boldsymbol{u}_{r}$-modules $V(\lambda)$ and $V(r, \lambda), \lambda \in P_{p^{r}}$, are isomorphic.

Proof. We have seen above in (9) that $V(\lambda)=\boldsymbol{u}_{r} v_{\lambda}$. Therefore it suffices to prove $V(\lambda)^{\operatorname{ker}\left(\gamma_{r}\right)}=K v_{\lambda}$. Write $N=V(\lambda)^{\operatorname{ker}\left(\gamma_{r}\right)}$. Because of $\boldsymbol{x}_{r} H \subseteq H \boldsymbol{x}_{r}$ and (12), $N=\oplus \sum_{\mu \in P(\lambda)}\left(V(\lambda)_{\mu} \cap N\right)$. Using the fact that $X=\left\langle\left\{X_{\alpha_{i}, c} \mid i=1, \ldots, l, c \in N\right\}\right\rangle$, 
Lemma 5 and the commutation rule $X \boldsymbol{x}_{\boldsymbol{r}}=\boldsymbol{x}_{\boldsymbol{r}} X$ we can see, as in [1], pp. 43 and 44, that $V(\lambda)_{\mu} \cap N=0$ for all $\mu \neq \lambda$. Hence $N=V(\lambda)_{\lambda}=K v_{\lambda}$.

Corollary. (i) $\operatorname{Irr}\left(\boldsymbol{u}_{1}\right) \subset \operatorname{Irr}\left(\boldsymbol{u}_{2}\right) \subset \ldots$

(ii) $\operatorname{Irr}\left(U_{K}\right)=\bigcup_{r=1}^{\infty} \operatorname{Irr}\left(\boldsymbol{u}_{r}\right)$.

Finally, we show how the irreducible $U_{K}$-module $V(\lambda)$ can be constructed as a tensor product of modules of the form $V\left(p^{k} \mu\right), k \in \boldsymbol{Z}^{+}, \mu \in P_{p}$.

Lemma 6. Let $r \in \boldsymbol{N}$ and $\lambda \in P_{p}$. Then $\boldsymbol{y}_{\boldsymbol{r}} V\left(p^{r} \lambda\right)=0$ and $\boldsymbol{x}_{\boldsymbol{r}} V\left(p^{r} \lambda\right)=0$.

Proof. Let $v_{\boldsymbol{r}}=v_{p^{r} \lambda}$ in which case $V\left(p^{r} \lambda\right)=K v_{\boldsymbol{r}} \oplus Y v_{\boldsymbol{r}}$. Since $\boldsymbol{y}_{\boldsymbol{r}} Y=Y_{\boldsymbol{y}_{\boldsymbol{r}}}$ and $\boldsymbol{y}_{\boldsymbol{r}}$ is generated by elements of the form $X_{-\alpha_{i}, a}, 1 \leqq i \leqq l, 0<a<p^{r}$, the assertion $\boldsymbol{y}_{\boldsymbol{r}} V\left(p^{r} \lambda\right)=0$ follows if $X_{-\alpha_{i}, a} v_{\boldsymbol{r}}=0$ for this $i, a$. But using induction on $a$, it can easily be proved that

$$
X_{\beta, c} X_{-\alpha_{i}, a} v_{r}=0 \text { for all } \beta \in \Delta, c \in N, i \in\{1, \ldots, l\}, \quad 0<a<p^{r} .
$$

Then $X_{-\alpha_{i}, a} v_{r} \in V\left(p^{r} \lambda\right)^{\operatorname{ker}(\gamma)} \cap Y v_{r}=0$, which proves the first claim.

According to (9) we may write $V\left(p^{r} \lambda\right)=K v_{\boldsymbol{r}} \oplus \boldsymbol{y}_{\boldsymbol{r}+\mathbf{1}} v_{\boldsymbol{r}}$. Since the elements $X_{-\alpha_{i}, p^{j}}, 1 \leqq i \leqq l, 0 \leqq j \leqq r$, generate $\boldsymbol{y}_{r+1}$ it is now clear that vectors

span $V\left(p^{r} \lambda\right)$. Hence

$$
v_{r}, X_{-\beta_{1}, p^{r}} \ldots X_{-\beta_{s}, p^{r}} v_{r}, \quad s \in N, \beta_{i} \in \Delta,
$$

$$
\boldsymbol{x}_{\boldsymbol{r}} V\left(p^{r} \lambda\right) \subseteq \boldsymbol{u}_{\boldsymbol{r}+1} \boldsymbol{x}_{\boldsymbol{r}} v_{\boldsymbol{r}}+\boldsymbol{u}_{\boldsymbol{r}+1} \boldsymbol{y}_{r} \boldsymbol{u}_{r+1} v_{\boldsymbol{r}}=0,
$$

because $\boldsymbol{x}_{\boldsymbol{r}} v_{\boldsymbol{r}}=0$ and $\boldsymbol{y}_{\boldsymbol{r}} V\left(p^{\boldsymbol{r}} \lambda\right)=0$.

Now $U_{K}$ has a natural Hopf algebra structure arising from the Hopf algebra ttructure of the universal enveloping algebra $U(\mathfrak{g})$. Let $\Delta$ denote the diagonalizasion map and set $\Delta_{1}=\Delta, \Delta_{n}=\left(\Delta \otimes I^{n-1}\right) \Delta_{n-1}, n \geqq 2$. This is determined explicitly by

$$
\begin{aligned}
& \Delta_{n-1}\left(X_{\alpha, a}\right)=\sum_{a_{1}+\ldots+a_{n}=a} X_{\alpha, a_{1}} \otimes \ldots \otimes X_{\alpha, a_{n}} \\
& \Delta_{n-1}\left(H_{i, b}\right)=\sum_{b_{1}+\ldots+b_{n}=b} H_{i, b_{1}} \otimes \ldots \otimes H_{i, b_{n}}
\end{aligned}
$$

for all $n \geqq 2, \alpha \in \Phi, 1 \leqq i \leqq l, a, b \in Z^{+}$. If $V_{1}, \ldots, V_{n}$ are left $U_{K}$-modules then so is $V_{1} \otimes \ldots \otimes V_{n}$, the action being given by $\Delta_{n-1}$.

Proposition 6. Write $\lambda \in P^{+}$in the form $\lambda=\lambda_{0}+p \lambda_{1}+\ldots+p^{k} \lambda_{k}$ where $\lambda_{i} \in P_{p}$. Then

$$
V(\lambda) \cong_{U_{K}} V\left(\lambda_{0}\right) \otimes V\left(p \lambda_{1}\right) \otimes \ldots \otimes V\left(p^{k} \lambda_{k}\right) .
$$

Proof. Let $M=V\left(\lambda_{0}\right) \otimes \ldots \otimes V\left(p^{k} \lambda_{k}\right)$ and $v_{i}=v_{p^{i} \lambda_{i}}, i=0, \ldots, k$. The assertion follows if we can show

and

$$
\begin{gathered}
M^{\operatorname{ker}\left(\gamma_{k+1}\right)}=K\left(v_{0} \otimes \ldots \otimes v_{k}\right), \\
M=\boldsymbol{u}_{k+1}\left(v_{0} \otimes \ldots \otimes v_{k}\right)
\end{gathered}
$$

$$
K\left(v_{0} \otimes \ldots \otimes v_{k}\right) \cong_{h_{k+1}} K v_{\lambda}
$$


Let $m=\sum_{i=1}^{n} w_{i} \otimes x_{i} \in M^{\mathrm{ker}\left(\gamma_{k+1}\right)}$ where each $w_{i} \in V\left(\lambda_{0}\right)$ and $x_{i} \in V\left(p \lambda_{1}\right) \otimes \ldots \otimes V\left(p^{k} \lambda_{k}\right)$. We may assume that the vectors $x_{1}, \ldots, x_{n}$ are linearly independent. If $\alpha \in \Phi^{+}$ Lemma 6 implies

$$
0=X_{\alpha, 1} m=\sum_{i=1}^{n} X_{\alpha, 1} w_{i} \otimes x_{i} .
$$

The linear independence of the vectors $x_{i}$ then forces $X_{\alpha, 1} w_{i}=0$ for all $i=1, \ldots, n$. Thus each $w_{i} \in V\left(\lambda_{0}\right)^{\operatorname{ker}\left(\gamma_{1}\right)}=K v_{0}$ and we get

$$
M^{\operatorname{ker}\left(\gamma_{k+1}\right)} \subseteq K v_{0} \otimes\left(V\left(p \lambda_{1}\right) \otimes \ldots \otimes V\left(p^{k} \lambda_{k}\right)\right)^{\operatorname{ker}\left(\gamma_{k+1}\right)} .
$$

Replacing $X_{\alpha, 1}$ by $X_{\alpha, p}$ in the above argument gives

$$
\left(V\left(p \lambda_{1}\right) \otimes \ldots \otimes V\left(p^{k} \lambda_{k}\right)\right)^{\operatorname{ker}\left(\gamma_{k+1}\right)} \subseteq K v_{1} \otimes\left(V\left(p^{2} \lambda_{2}\right) \otimes \ldots \otimes V\left(p^{k} \lambda_{k}\right)\right)^{\operatorname{ker}\left(\gamma_{k+1}\right)} .
$$

Continuing this process leads to $M^{\mathrm{ker}\left(\gamma_{k+1}\right)}=K\left(v_{0} \otimes \ldots \otimes v_{k}\right)$.

Let

$$
N_{j}=\left\{1, X_{-\beta_{1}, p^{j}} \ldots X_{-\beta_{s,} p^{j}} \mid s \in N, \beta_{i} \in \Delta, i=1, \ldots, s\right\}
$$

where $j=0, \ldots, k$. The set $\left\{x_{0} v_{0} \otimes \ldots \otimes x_{k} v_{k} \mid x_{j} \in N_{j}, j=0, \ldots, k\right\}$ is seen to span $M$ as a vector space (cf. (13)). But Lemmas 4 and 6 imply $\left(x_{0} \ldots x_{k}\right)\left(v_{0} \otimes \ldots \otimes v_{k}\right)=$ $x_{0} v_{0} \otimes \ldots \otimes x_{k} v_{k}$, hence $M=\boldsymbol{u}_{k+1}\left(v_{0} \otimes \ldots \otimes v_{k}\right)$.

Finally, it can easily be confirmed that

$$
H_{i, b}\left(v_{0} \otimes \ldots \otimes v_{k}\right)=\varphi_{\lambda}\left(H_{i, b}\right)\left(v_{0} \otimes \ldots \otimes v_{k}\right) \quad \text { for all } 1 \leqq i \leqq l, b \in \boldsymbol{Z}^{+} .
$$

Therefore $K\left(v_{0} \otimes \ldots \otimes v_{k}\right) \cong{ }_{h_{k+1}} K v_{\lambda}$ and the proof is complete.

5. Irreducible representations of the universal Chevalley group. Let $G=$ $\left\langle x_{\alpha}(t) \mid \alpha \in \Phi, t \in K\right\rangle$ be the universal Chevalley group of type $\mathfrak{g}$ over the field $K$ ([4], p. $161)$. Let $K_{p^{r}} \subset K(r \in N)$ be a finite field of order $p^{r}$ and $G_{r}=\left\langle x_{\alpha}(t) \mid \alpha \in \Phi, t \in K_{p^{r}}\right\rangle$ the corresponding finite subgroup of $G$. The irreducible rational $G$-modules are known to correspond one-to-one to irreducible $U_{K}$-modules and similarly for irreducible $G_{\boldsymbol{r}}$ - and $\boldsymbol{u}_{\boldsymbol{r}}$-modules. Thus

$$
\begin{gathered}
\operatorname{Irr}(G)=\operatorname{Irr}\left(U_{K}\right)=\left\{[V(\lambda)] \mid \lambda \in P^{+}\right\}, \\
\operatorname{Irr}\left(G_{r}\right)=\operatorname{Irr}\left(\boldsymbol{u}_{r}\right)=\left\{[V(\lambda)] \mid \lambda \in P_{p^{r}}\right\}
\end{gathered}
$$

and the groups $G, G_{r}$ act on the modules $V(\lambda)=W(\lambda)^{\prime}$ according to the rule

$$
x_{\alpha}(t) v=\sum_{n \geqq 0} t^{n} X_{\alpha, n} v \quad \text { for all } \quad \alpha \in \Phi, t \in K, v \in V(\lambda) .
$$

This shows how the irreducible rational $G$-modules and irreducible $G_{r}$-modules over $K$ can all be obtained starting from one-dimensional $H$-modules (resp. $\boldsymbol{h}_{r}$-modules) lifting them up to $U_{K}$-modules (resp. $\boldsymbol{u}_{r}$-modules) as in $\S 2$ and then transforming them into $G$-modules (resp. $G_{r}$-modules) using (14).

Let $\lambda \in P_{p}$ and $k \in Z^{+}$. Then $V\left(p^{k} \lambda\right) \cong{ }_{G} V(\lambda)^{\left(p^{k}\right)}$ where $V(\lambda)^{\left(p^{k}\right)}=V(\lambda)$ with $G$-action given by $x_{\alpha}(t) v=x_{\alpha}\left(t^{p^{k}}\right) v, \alpha \in \Phi, t \in K, v \in V(\lambda)$ ([11], p. 217). If the tensor 
product of $U_{K}$-modules in Proposition 6 is viewed as a $G$-module it becomes an ordinary tensor product of $G$-modules $V\left(\lambda_{0}\right), \ldots, V\left(p^{k} \lambda_{k}\right)$. Hence as a corollary to Proposition 6 one obtains a new proof of Steinberg's tensor product theorem:

Proposition 7. Let $\lambda=\lambda_{0}+p \lambda_{1}+\ldots+p^{k} \lambda_{k}$ where $\lambda_{i} \in P_{p}$. Then $V(\lambda) \cong{ }_{G} V\left(\lambda_{0}\right) \otimes$ $V\left(\lambda_{1}\right)^{(p)} \otimes \ldots \otimes V\left(\lambda_{k}\right)^{\left(p^{k}\right)}$.

\section{References}

[1] Borel, A.: Properties and linear representations of Chevalley groups. - Seminar on Algebraic Groups and Related Finite Groups, Lecture Notes in Mathematics 131, SpringerVerlag, Berlin-Heidelberg-New York, 1970.

[2] Cline, E., B. Parshall, and L. Scott: Cohomology, hyperalgebras and representations. - To appear.

[3] CuRTis, C. W., and I. ReINER: Representation theory of finite groups and associative algebras. Interscience Publishers, New York-London, 1962.

[4] Humphreys, J. E.: Introduction to Lie algebras and representation theory. - Springer-Verlag, New York-Heidelberg-Berlin, 1972.

[5] Humphreys, J. E.: Ordinary and modular representations of Chevalley groups. - Lecture Notes in Mathematics 528, Springer-Verlag, Berlin-Heidelberg-New York, 1976.

[6] Humphreys, J. E.: On the hyperalgebra of a semisimple algebraic group. - Contributions to Algebra, A collection of papers dedicated to Ellis Kolchin, edited by H. Bass, P. J. Cassidy, and J. Kovacic, Academic Press, New York-San Francisco-London, 1977, 203-210.

[7] JACOBson, N.: Lie algebras. - Interscience Publishers, New York-London, 1962.

[8] JANTZEN, J. C.: Darstellungen halbeinfacher algebraischer Gruppen und zugeordnete kontravariante Formen. - Bonn. Math. Schr. 67, 1973, 1-124.

[9] Neuvonen, T.: A criterion for complete reducibility of restricted Lie modules. - Arch. Math. (Basel) 28, 1977, 149-156.

[10] Neuvonen, T.: A complete reducibility criterion with an application to representations of semisimple groups. - J. Algebra (to appear).

[11] Steinberg, R.: Lectures on Chevalley groups. - Yale University, 1967.

[12] Sweedler, M. E.: Hopf algebras. - W. A. Benjamin, Inc., New York, 1969.

[13] Wallach, N.: Induced representations of Lie algebras and a theorem of Borel-Weil. - Trans. Amer. Math. Soc. 136, 1969, 181-187.

[14] Wallach, N.: Induced representations of Lie algebras II. - Proc. Amer. Math. Soc. 21, 1969, $161-166$.

University of Turku

Department of Mathematics

SF-20500 Turku 50

Finland

Received 7 May 1979 